\title{
MicroRNA profiling and their pathways in South African individuals with prediabetes and newly diagnosed type 2 diabetes mellitus
}

\author{
Tandi E. Matsha ${ }^{1}$, Andre P. Kengne ${ }^{2,3}$, Stanton Hector ${ }^{1}$, Desiree L. Mbu ${ }^{1}$, Yandiswa \\ Y. Yako ${ }^{4}$ and Rajiv T. Erasmus ${ }^{5}$ \\ ${ }^{1}$ Department of Biomedical Sciences, Faculty of Health and Wellness Science, Cape Peninsula University of Technology, Cape \\ Town, South Africa \\ ${ }^{2}$ Non-Communicable Diseases Research Unit, South African Medical Research Council, Cape Town, South Africa \\ ${ }^{3}$ Department of Medicine, University of Cape Town, Cape Town, South Africa \\ ${ }^{4}$ Department of Human Biology, Faculty of Health Sciences, Walter Sisulu University, Mthatha, South Africa \\ ${ }^{5}$ Department of Pathology, Faculty of Health Sciences, National Health Laboratory Service (NHLS) and University of \\ Stellenbosch, Cape Town, South Africa \\ Correspondence to: Tandi E. Matsha, email: matshat@cput.ac.za, tandimatsha@gmail.com \\ Keywords: Africa; diabetes; miRNA; prediabetes; sequencing \\ Received: August 18, $2017 \quad$ Accepted: April 06, $2018 \quad$ Published: July 17, 2018 \\ Copyright: Matsha et al. This is an open-access article distributed under the terms of the Creative Commons Attribution License \\ 3.0 (CC BY 3.0), which permits unrestricted use, distribution, and reproduction in any medium, provided the original author and \\ source are credited.
}

\section{ABSTRACT}

Early identification of individuals with elevated risk of developing diabetes mellitus, followed by the implementation of effective prevention interventions can delay the onset of the disease and related complications. In this regard, recent studies have shown that miRNAs are useful as early markers of certain disease types, including diabetes. We used high throughput sequencing to assess miRNA expression profiles from whole blood of 12 individuals with screen-detected diabetes, 12 with prediabetes and 12 with normal glucose tolerance, matched for age, blood pressure, smoking and body mass index. We identified a total of 261 (57 novel) differentially expressed miRNA profiles between the study groups. Comparison of the miRNA expression profiles between prediabetess and diabetes revealed 25 common miRNA, but highlighted some interesting differences. For instance, three miRNAs ( $m i R-126-3 p, m i R-28-3 p$ miR-486-5p) were dysregulated in prediabetes compared to screen-detected diabetes. Target gene analysis showed thousands of potential genes and KEGG pathway analysis revealed 107 significant pathways of which some are involved signal transduction, cell-cell communications, cell growth and death, immune response, endocrine system and metabolic diseases. This first detailed African study has shown both known and novel differentially expressed miRNAs in relation to glucose tolerance.

\section{INTRODUCTION}

Diabetes mellitus (DM) is a major cause of morbidity and mortality worldwide and has resulted in deaths of approximately five million people aged between 20 and 79 years in 2015 according to the International diabetes federation [1]. Sub-Saharan Africa (SSA) is currently experiencing the fastest relative growth of people with diabetes that remains undiagnosed in many [2]. In 2015, the International Diabetes Federation (IDF) Diabetes Atlas, estimated that nearly $2 / 3^{\text {rd }}$ of the 14.2 million sub-Saharan Africans adults with diabetes were undiagnosed [1]. Overt DM is often preceded by prediabetes, a term used to define subjects with either or both impaired fasting glucose (IFG) and impaired glucose tolerance (IGT). Approximately, $5-10 \%$ of individuals with prediabetes develop diabetes annually with up to $70 \%$ eventually progressing to the stage of the disease [3, 4]. Lifestyle modifications or drug 
interventions can delay the onset of DM $[5,6]$, however this is dependent on the identification of those at risk of progression to overt DM. This identification is heavily dependent on the diagnostic criteria which are continually revised and vary across stakeholders. For example, the World Health organisation (WHO) and American Diabetes Federation differ in the description of IFG, respectively $6.1-6.9 \mathrm{mmol} / /$ and $5.6-6.9 \mathrm{mmol} / \mathrm{L}$. Moreover in 2010 the ADA included $\mathrm{HbA} 1 \mathrm{c}$ ranging from $5.7 \%$ to $6.4 \%$ as diagnostic criterion for prediabetes. These realities validate the critical need for earlier and more efficient biomarkers for diabetes risk screening. In this regard, recent studies [79] have shown that micro RNAs (miRNAs) are useful as early markers of certain disease types, including diabetes.

To date, over 1881 miRNA precursors that can generate over 2588 mature miRNAs have been identified in the human genome (www.mirbase.org) which control $\sim 50 \%$ of all mammalian protein-coding genes [10]. miRNAs are a class of small ( $\sim 19-24$ nucleotides in length), endogenous, evolutionarily conserved RNAs that function as posttranscriptional regulators of gene expression [1113]. They primarily function by binding to complementary target sequences in messenger RNA (mRNA) and by interfering with the translational machinery, thereby prevent or alter the production of the protein product [11-13]. In addition to repressing translation, miRNA binding to its target mRNA also triggers the recruitment and association of mRNA decay factors, leading to mRNA destabilization, degradation, and resultant decrease in expression levels [13]. Dysregulated expression of miRNAs is associated with the pathogenesis of a variety of diseases including dysglycaemia and vascular complications. Several miRNAs that are involved in the regulation of insulin signaling, lipid and glucose homeostasis have been identified in various tissues $[14,15]$, however, findings are inconsistent owing to techniques, sample and genetic diversity of populations studied $[16,17]$.

Despite the growing evidence of the important role and potential diagnostic value of miRNAs in dysglycaemia, such properties are yet to be demonstrated in the African setting. Therefore, in the present study we aimed to identify dysregulated miRNA in a South African mixed ancestry population previously reported to be at high risk of diabetes [18]. To avoid potential bias from treatment induced alterations in miRNA expression, we focused on individuals with normal glucose tolerance (NGT), prediabetes individuals with IGT only and those with screen-detected diabetes who had not initiated glucose lowering drug treatment.

\section{RESULTS}

\section{General characteristics of participants}

The characteristics of participants overall, and according to glucose tolerance status are summarised in
Table 1. All participants were female and the mean levels of diastolic blood pressure, systolic blood pressure, low density lipoprotein cholesterol, high density lipoprotein cholesterol, age, body mass index, and serum cotinine did not differ significantly by status for glucose tolerance (all $\mathrm{p} \geq 0.06$ ).

\section{miRNA expression profiling}

All 36 samples (12 NGT, 12 IGT and 12 screendetected diabetes) met the quality control standards. The clean reads (Adapter-trimmed reads) met and exceeded the specified sequencing coverage of $5 \mathrm{M}$ (Supplementary Table 1). We generated Heat Map and Unsupervised Hierarchical Clustering on all expressed miRNAs (at least expressed in one sample) to produce miRNA or condition trees to allow us to pick out groups of similar miRNA. The result of hierarchical clustering on conditions showed a distinguishable miRNA expression profile among samples (Figure 1). For the identification of differentially expressed miRNAs, we computed "fold change" (i.e. the ratio of the group averages) and p-value between each group. miRNAs having fold changes $\geq 1.3$, p-value $\leq 0.1$ were selected as the differentially expressed miRNAs. Based on pre-specified criteria, we then used volcano plots to visualize the significantly differentially expressed pre-miRNAs between the study groups (Figure 2 ). In total, we identified a total of 261 significant premiRNAs that generated 237 mature miRNAs at varying levels of expression. Upregulated pre-miRNA (mature miRNA) were 117 (107) between screen-detected DM versus NGT, 58 (54) between IGT and NGT and 56 (50) between screen-detected DM and IGT, and downregulated miRNAs were respectively, 3 (2); 9 (7) and 18 (17). Fifty seven $(22 \%)$ of the differentially expressed miRNAs were novel, of which 21 were upregulated in DM versus NGT, 19 in IGT versus NGT and 8 in DM versus IGT, whilst none were downregulated in DM versus NGT, 1 in IGT versus NGT and 8 in DM versus IGT. The details of the ten most differentially expressed miRNAs can be found in Supplementary Table 2 which shows that miR-15b-5p remained amongst the ten most differentially expressed miRNA when screen-detected diabetes was compared to either NGT or IGT. Among the down-regulated species, miR-486-5p was found in both screen-detected DM versus NGT and screen-detected DM versus IGT. A total of 28 pre-miRNA encoding 25 matured miRNAs were upregulated in both screen-detected DM and IGT when compared to NGT (Table 2). Among the ten most differentially expressed miRNAs in IGT versus NGT, eight (miR-99b-5p, -30e-3p, -1260a, -4443, -novel-chr2_50989, $-1260 \mathrm{~b},-151 \mathrm{a}-5 \mathrm{p},-\mathrm{miR}-223-3)$ were also dysregulated in screen-detected DM when compared to NGT. Two of these, miR-novel-chr2_50989 and miR-223-3p were also found to be amongst the top ten differentially expressed miRNAs between screen-detected DM versus NGT. 
Table 1: Characteristics of the 36 women included overall, and by status for glucose tolerance

\begin{tabular}{|c|c|c|c|c|c|}
\hline Characteristics & Total & Normal & IGT & New DM & p-value \\
\hline $\mathrm{n}$ & 36 & 12 & 12 & 12 & \\
\hline Age (years) & $53.4(7.8)$ & $52.1(7.8)$ & $53.5(8.5)$ & $54.8(7.5)$ & 0.717 \\
\hline Weight (kg) & $76.8(19.7)$ & $70.2(18.9)$ & $79.3(21.7)$ & $80.8(18.3)$ & 0.370 \\
\hline Height $(\mathrm{cm})$ & $155.8(6.5)$ & $157.2(7.9)$ & $154.4(6.0)$ & $156.1(5.9)$ & 0.600 \\
\hline $\begin{array}{l}\text { Body mass index } \\
\left(\mathrm{kg} / \mathrm{m}^{2}\right)\end{array}$ & $31.5(8.4)$ & $27.3(5.8)$ & $33.3(9.1)$ & $33.5(8.9)$ & 0.141 \\
\hline $\begin{array}{l}\text { Waist } \\
\text { circumference } \\
(\mathrm{cm})\end{array}$ & $93.9(18.7)$ & $83.2(18.5)$ & $97.1(13.8)$ & $101.3(19.7)$ & 0.041 \\
\hline $\begin{array}{l}\text { Hip } \\
\text { circumference } \\
(\mathrm{cm})\end{array}$ & $107.1(17.7)$ & $102.0(18.4)$ & $109.9(18.6)$ & $109.4(16.6)$ & 0.487 \\
\hline Waist/hip ratio & $0.87(0.08)$ & $0.81(0.07)$ & $0.89(0.06)$ & $0.92(0.07)$ & 0.001 \\
\hline $\begin{array}{l}\text { Systolic blood } \\
\text { pressure (mmHg) }\end{array}$ & $138.4(27.5)$ & $135.3(30.8)$ & $137.0(18.5)$ & $142.9(32.9)$ & 0.784 \\
\hline $\begin{array}{l}\text { Diastolic blood } \\
\text { pressure }(\mathrm{mmHg})\end{array}$ & $87.0(18.0)$ & $78.0(16.1)$ & $88.9(11.7)$ & $94.2(22.1)$ & 0.077 \\
\hline Pulse rate (bpm) & $72.1(13.5)$ & $64.8(12.2)$ & $71.3(5.8)$ & $80.1(16.4)$ & 0.016 \\
\hline $\begin{array}{l}\text { Fasting plasma } \\
\text { glucose } \\
(\mathrm{mmol} / \mathrm{L})\end{array}$ & $6.3(2.9)$ & $4.6(0.8)$ & $5.3(0.5)$ & $9.1(3.6)$ & $<0.0001$ \\
\hline $\begin{array}{l}\text { 2-hour glucose } \\
(\mathrm{mmol} / \mathrm{L})\end{array}$ & $10.5(5.5)$ & $5.3(1.6)$ & $9.3(0.9)$ & $16.5(4.7)$ & $<0.0001$ \\
\hline $\begin{array}{l}\text { Fasting insulin } \\
(\mathrm{mIU} / \mathrm{L})^{*}\end{array}$ & $8.10(5.70-12.50)$ & $5.65(2.95-7.85)$ & $9.45(7.55-12.50)$ & $14.10(5.90-21.20)$ & 0.0058 \\
\hline $\begin{array}{l}\text { 2-hour Insulin } \\
(\mathrm{mIU} / \mathrm{L})^{*}\end{array}$ & $53.20(29.20-97.90)$ & $28.50(17.80-40.40)$ & $\begin{array}{c}111.20(60.20- \\
187.20)\end{array}$ & $48.80(31.45-83.30)$ & 0.0044 \\
\hline $\begin{array}{l}\text { Triglycerides } \\
(\mathrm{mmol} / \mathrm{L})\end{array}$ & $1.6(1.1)$ & $1.0(0.3)$ & $1.76(1.0)$ & $2.2(1.3)$ & 0.023 \\
\hline $\begin{array}{l}\text { LDL Cholesterol } \\
\text { (Measured) } \\
(\mathrm{mmol} / \mathrm{L})\end{array}$ & $3.6(1.0)$ & $3.3(1.0)$ & $3.5(1.0)$ & $4.2(0.8)$ & 0.060 \\
\hline $\begin{array}{l}\text { HDL cholesterol } \\
(\mathrm{mmol} / \mathrm{L})\end{array}$ & $1.4(0.5)$ & $1.6(0.5)$ & $1.3(0.4)$ & $1.4(0.6)$ & 0.451 \\
\hline $\begin{array}{l}\text { Total cholesterol } \\
(\mathrm{mmol} / \mathrm{L})\end{array}$ & $5.7(1.0)$ & $5.5(1.1)$ & $5.4(1.0)$ & $6.4(0.8)$ & 0.043 \\
\hline $\begin{array}{l}\text { Total/HDL } \\
\text { cholesterol ratio }\end{array}$ & $4.3(1.4)$ & $3.7(1.2)$ & $4.3(1.3)$ & $5.02(1.4)$ & 0.077 \\
\hline $\begin{array}{l}\text { High-sensitivity } \\
\text { CRP (mg/L) }\end{array}$ & $7.84(8.56)$ & $3.12(2.73)$ & $9.28(5.93)$ & $11.11(12.34)$ & 0.052 \\
\hline $\begin{array}{l}\text { Cotinine (ng/ } \\
\mathrm{mL}^{*}\end{array}$ & $22.5(10.0-220.5)$ & $22.5(10.0-261.0)$ & $91.0(10.0-220.0)$ & $85.0(10.0-206.0)$ & 1.0000 \\
\hline
\end{tabular}

*, median $\left[25^{\text {th }}-75^{\text {th }}\right.$ percentiles), and p-values from Kruskal wallis test. 

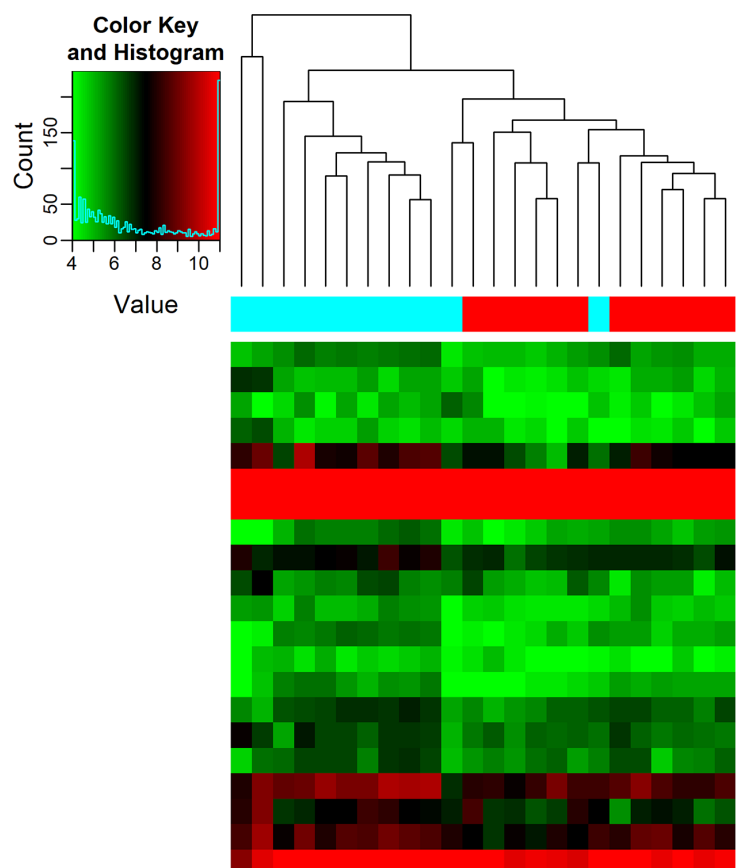

hsa-miR-novel-chr8_81058_hsa-mir-novel-chr8_8105 hsa-miR-novel-chr6_71512_hsa-mir-novel-chr6_71512 hsa-miR-novel-chr1_36178_hsa-mir-novel-chr1_36178 hsa-miR-novel-chr1_40444_hsa-mir-novel-chr1_40444 hsa-miR-4443_hsa-mir-4443

hsa-miR-15b-5p_hsa-mir-15b

hsa-miR-140-3p_hsa-mir-140

hsa-miR-novel-chr19_33126_hsa-mir-novel-chr19_33126 hsa-miR-28-3p_hsa-mir-28

hsa-miR-novel-chr5_67266_hsa-mir-novel-chr5_67266 hsa-miR-330-5p hsa-mir-330

hsa-miR-5480-3p_hsa-mir-5480-2

hsa-miR-4646-5p_hsa-mir-4646

hsa-mir-novel-chr22_48686_hsa-mir-novel-chr22_48686

hsa-miR-181a-2-3p_hsa-mir-181a-2

hsa-miR-378i_hsa-mir-378i

nea-mir-599-5p nae-mir-509

hsa-miR-3158-3p_hsa-mir-3158-1

hsa-miR-novel-chr2 _55842 hsa-mir-novel-chr2__55842

hsa-miR-1307-3p__na-mir-1307

hsa-miR-novel-chr20_44211_hsa-mir-novel-chr20_44211

hsa-miR-99b-3p_hsa-mir-99b

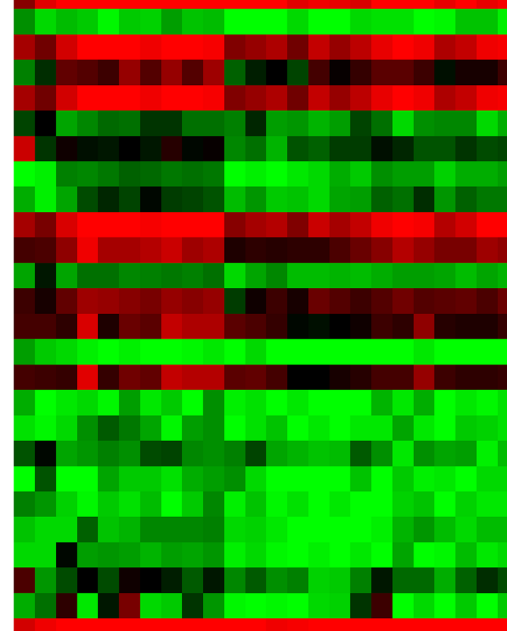

h⿻

hsa-miR-103a-3p_hsa-mir-103a-1

hsa-miR-novel-chr5_67265_hsa-mir-novel-chr5_67265

hsa-miR-99b-5p_hsa-mir-99b

hsa-miR-5480-3p hsa-mir-548

hsa-miR-novel-chr7_ 78559 _hsa-mir-novel-chr7__78559

hsa-miR-novel-chr5_70692_hsa-mir-novel-chr5_70692

hsa-miR-584-5p_hsa-mir-584

hsa-miR-novel-chr11_6801_hsa-mir-novel-chr11_6801

hsa-miR-425-5p_hsa-mir-425

hsa-miR-1260a hsa-mir-1260a

hsa-miR-139-3p hsa-mir-139

hsa-miR-1260

tha-min-1200

hsa-miR-504-5p_hsa-mir-504

hsa-miR-151b_hsa-mir-151b

hsa-miR-novel-chr13 13520 hsa-mir-novel-chr13 13520

hsa-miR-1270_hsa-mir-1270

hsa-miR-1908-5p hsa-mir-1908

hsa-miR-novel-chr17_28671_hsa-mir-novel-chr17_28671

hsa-miR-novel-chr2_50989_hsa-mir-novel-chr2_50989

hsa-miR-30e-3p_hsa-mir-30e

hsa-miR-1299_hsa-mir-1299

hsa-miR-181a-5p_hsa-mir-181a-1

hsa-miR-181a-5p_hsa-mir-181a-2

hsa-miR-378a-3p_hsa-mir-378a

hsa-miR-3158-3p_hsa-mir-3158-2

hsa-miR-151a-3p_hsa-mir-151a

hsa-miR-151a-5p hsa-mir-151

hsa-miR-339-3p hsa-mir-339

hsa-mir-novel-chrX_90454 hsa-mir-novel-chrX_90454

hsa-miR-126-3p_hsa-mir-126

hsa-miR-378d_hsa-mir-378d-1

hsa-miR-3615_hsa-mir-3615

hsa-miR-novel-chr13_13519_hsa-mir-novel-chr13_13519

hsa-miR-novel-chr5_68297_hsa-mir-novel-chr5_68297

hsa-miR-92b-3p_hsa-mir-92b

hsa-miR-4467_hsa-mir-4467

hsa-miR-novel-chr21_47609_hsa-mir-novel-chr21_47609

hsa-miR-4433b-5p_hsa-mir-4433b

hsa-miR-1255b-5p_hsa-mir-1255b-2

hsa-miR-92a-3p_hsa-mir-92a-2

hsa-miR-92a-3p_hsa-mir-92a-1

hsa-miR-4685-3p_hsa-mir-4685

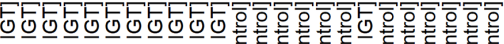

\%ำ

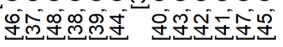

(Continued) 

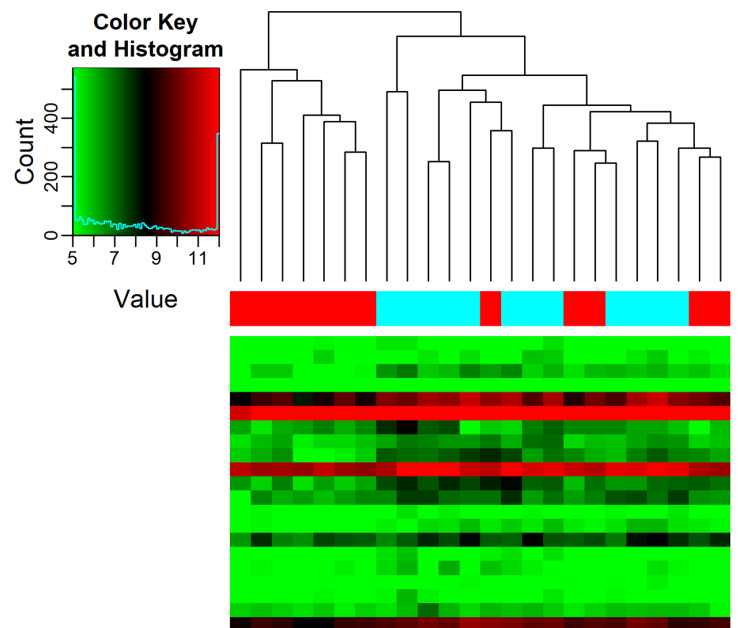

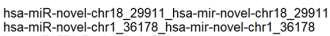

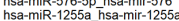

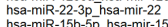

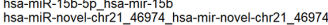

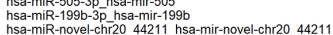

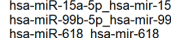

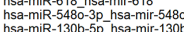

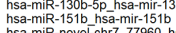

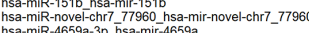

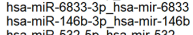

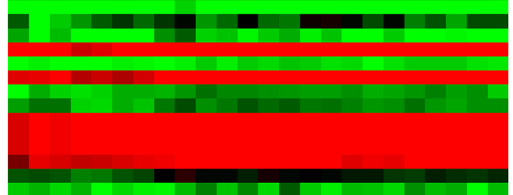

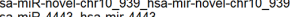

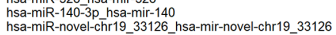

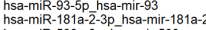

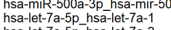

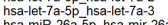

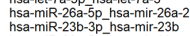

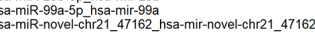

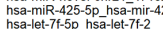

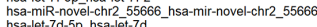

hsa-mia-26a-5p hsa-mir-26a-1

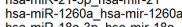

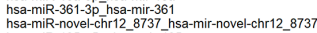

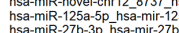

hsa-miR-191-5p hsa-mir-191

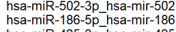

hsa-miR-novel-chr18_30554 hsa-mir-novel-chr18_30554

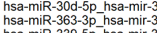

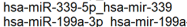

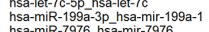

hsa-miR-1466-5p_hsa-mir-14

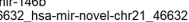

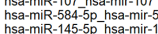

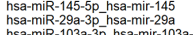

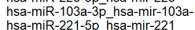

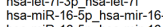

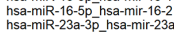

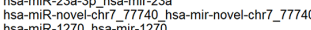

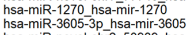

hsa-mir-novel-chr2_-50989

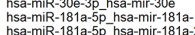

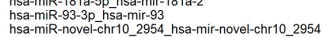

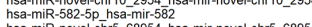

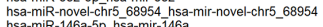

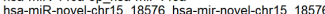

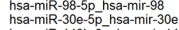

hsa-miR-1486-3b hsa-mir-148

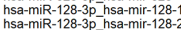

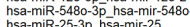

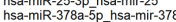

hsa-miR-10-5p- hsa-mir-10a
hsa-miR--novel-chrr17 25546 hsa-mir-novel-chr17_25546

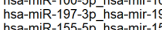

hsa-mii-26-5-5p hsa-miri-26

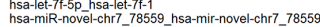

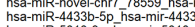

his

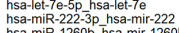

hsa-miR-novel-chr17_28671-hsa-mir-novel-chr1__28671

hsa-miR-1306-5p hsa-mir-130

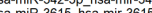

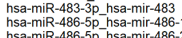

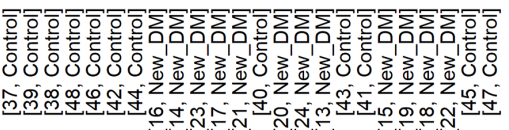

(Continued) 

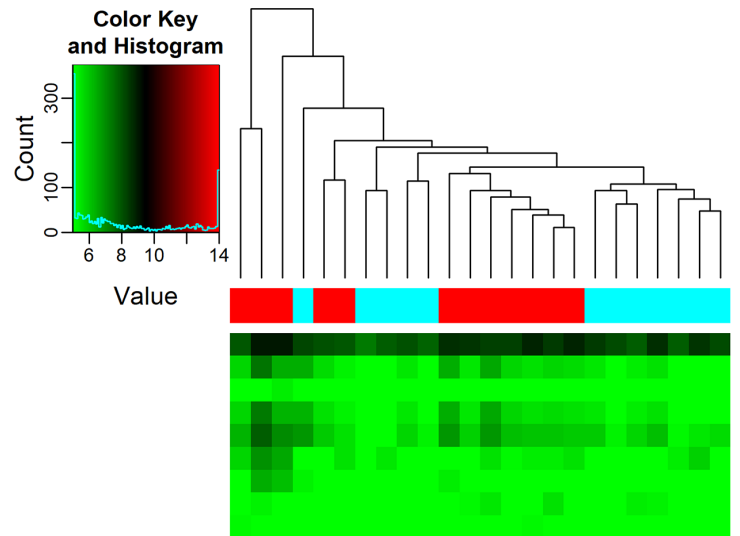

hsa-miR-744-5p_hsa-mir-744

hsa-miR-novel-chr5 67266 hsa-mir-novel-chr5 67266 hsa-miR-139-3p hsa-mir-139

hsa-miR-novel-chr13 13520 hsa-mir-novel-chr13 13520 hsa-miR-novel-chr13_13519_hsa-mir-novel-chrr13_13519 hsa-miR-342-5p_hsa-mir-342

hsa-miR-novel-chr1_40444_hsa-mir-novel-chr1_40444 hsa-miR-5189-3p_hsa-mir-5189 hsa-miR-novel-chr7_76196_hsa-mir-novel-chr7_7619 hsa-miR-151a-3p_hsa-mir-151a

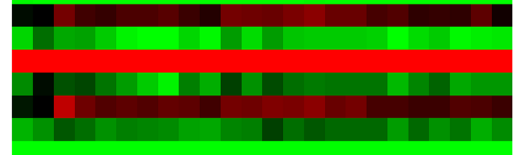
isa-miR-novel-chro_67265 hsa-mir-novel-chr5_6726 hsa-miR-486-5p_hsa-mir-486-2 hsa-miR-novel-chr2_55842_hsa-mir-novel-chr2__55842 hsa-miR-126-3p_hsa-mir-126 hsa-miR-28-3p_hsa-mir-28 hsa-miR-4516 hsa-mir-4516 hsa-miR-novel-chr5_68297_hsa-mir-novel-chr5_68297 hsa-miR-486-5p hsa-mir-486-1 hsa-miR-454-3p-hse-mir-454 has hsamir $300 .-5 p_{1}$ hsa-miR-1966-5p_hsa-mir-1960 hsa-miR-92b-3p_hsa-mir-92b hsa-miR-146a-Sp_hsa-mir-146a hsa-mir-novel-chr15_18576 hsa-miR-98-5p_hsa-mir-98 hsa-mir-22-3p_hsa-mir-22 hsa-miR-199a-3p_hsa-mir-199a-2 hsa-miR-199a-3p_hsa-mir-199a-1 hsa-miR-15b-5p_hsa-mir-15b hsa-miR-92a-3p_hsa-mir-92a-1 hsa-miR-345-5p_hsa-mir-345 hsa-miR-7976_hsa-mir-7976 hsa-miR-1306-5p_hsa-mir-1306 hsa-let-7i-5p_hsa-let-7i hsa-miR-107 hsa-mir-107 hsa-miR-378a-5p hsa-mir-378a hsa-miR-1996-3p hsa-mit-1995 hsa-miR-505-5p_hsa-mir-505

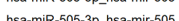

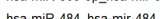
hsa-miR-48_-186-5p_hsa-mir-18 hsa-miR-186-5p_hsa-mir-186

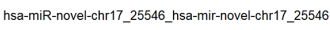
hsa-mir-4732-5p_hsa-mir-4732 hsa-miR-155-5p_hsa-mir-155 hsa-let-7a-5p_hsa-let-7a-1 hsa-let-7a-5p_hsa-let-7a-2 hsa-let-7a-5p_hsa-let-7a-3 hsa-miR-26a-5p_hsa-mir-26a-2 hsa-let-7f-5p_hsa-let-7f-1 hsa-let-7f-5p_hsa-let-7f-2 hsa-miR-3940-3p_hsa-mir-3940 hsa-miR-4433b-5p hsa-mir-4433b hsa-miR-5010-3p_hsa-mir-5010 hsa-miR-1255b-5p_hsa-mir-1255b-2 hsa-let-7g-5p_hsa-let-7g hsa-miR-1255b-5p_hsa-mir-1255b-1 hsa-miR-novel-chr2_55666_hsa-mir-novel-chr2__55666 hsa-let-7d-5p_hsa-let-7d hsa-miR-26a-5p_hsa-mir-26a-1 hsa-miR-26b-5p_hsa-mir-26b hsa-miR-26b-5p_hsa-mir-26b hsa-miR-novel-chr__ 77740 hssa hsa-miR-130b-5p_hsa-mir-130
hsa-miR-185-3p_hsa-mir-185 hsa-miR-185-3p_hsa-mir-185 hsa-miR-novel-chr7_77960_hsa-mir-novel-chr7_77960 hsa-mir-novel-chrif__2436__hsa-mir-novel-chr16_24365 hsa-miR-3605-3p_hsa-mir-3605 hsa-miR-4306_hsa-mir-430 hsa-miR-326_hsa-mir-326 hsa-miR-191-5p_hsa-mir-191 hsa-miR-novel-chr21_47609_hsa-mir-novel-chr21_47609 hsa-miR-6833-3p_hsa-mir-6833 hsa-miR-425-3p_hsa-mir-425

hsa-miR-novel-chr7_ 79616 hsa-mir-novel-chr7_ 79616 hsa-miR-4685-3p_hsa-mir-4685

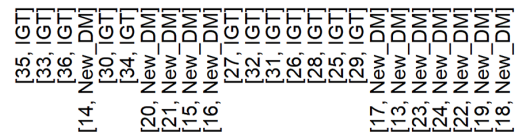

Figure 1: Differential miRNA expression in glucose tolerance groups. The heatmap shows all differentially expressed miRNAs at adjusted p-value $<0.05$. A. IGT versus NGT; B. screen-detected diabetes versus NGT; C. IGT versus screen-detected diabetes. Signal intensity was expressed as the $\log 2$ ratio between groups. Samples were grouped using hierarchical clustering based on similar expression profiles. Red, under-expression, white, no change, blue, overexpression. 


\section{Quantitative real time PCR of MicroRNAs}

As shown in Figure 3, we confirmed up-regulation of let7e-5p, let7f-5p, miR-15b-5p, miR-99b-5p, miR$103 a-3 p$ in subjects with diabetes. We also confirmed the non-expression of let7e-5p, let7f-5p in individuals with IGT.

\section{Target prediction and functional enrichment analysis}

We employed three databases namely, Mirbase, Miranda and Targetscan to conduct the analyses to improve the accuracy of our target site predictions. The target genes identified by the three databases were 690 in the screen-detected DM versus NGT, 535 screendetected DM versus IGT, and 320 in IGT versus NGT. The predicted target sites of dysregluted miRNAs are shown in the Venn diagrams (Figure 4). The KEGG pathway analysis demonstrated 107 pathways (Supplementary Table 3). These pathways include those associated with signal transduction, cell-cell communications, cell growth and death, immune response, endocrine system and metabolic diseases.

\section{DISCUSSION}

In this study, we employed high throughput sequencing to identify differentially expressed miRNAs associated with IGT and untreated diabetes in whole blood of South African mixed ancestry women, which in an earlier study we had established a high prevalence of undiagnosed IGT and DM [18). We observed evidence for differential expression of 61 in IGT, 109 in screendetected diabetes both when compared to individuals with normal glucose tolerance, of which 25 were common in both conditions. Although several of these dysregulated miRNAs have been linked to diabetic and non-diabetic hyperglycaemia, we also uncovered 57 novel miRNAs. Of note is hsa-miR-novel-chr2_50989 which had the highest fold change in screen-detected DM and remained in the top ten differentially expressed miRNAs in IGT. Functional annotation of genes that are potentially regulated by the miRNAs implicated showed that signal transduction pathways (PI3K-Akt, MAPK, HIF-1, cAMP, FoxO, ErbB, Ras, Rap1 and insulin resistance); carbohydrate metabolism; glycan biosynthesis and metabolism, cell communication, cell growth and death; immune system; endocrine system and metabolic diseases are likely involved in the development of hyperglycaemia in this population.

Successful diabetes prevention implementation strategies rely on accurate identification of people with prediabetes, who are likely to progress to the full stage of time. To this end, the diagnosis of IGT for instance, requires individuals to be subjected to the cumbersome oral glucose overload and measurement of blood glucose post 2 hours $[19,20]$. Consequently, individuals with this category of prediabetes largely remain undiagnosed. However, the emerging evidence for the role of miRNAs in the development of human diseases including diabetes is encouraging. In the development of diabetes, miRNAs have been reported to regulate metabolism, adipocyte differentiation, pancreatic development, $\beta$-cell mass, insulin biosynthesis, secretion, and signaling [21-25], underscoring their important role in the early diagnosis, pathogenesis and treatment of diabetes.

Some of the dysregulated miRNAs found in our study corroborate findings of many other studies that have aimed to characterize miRNAs in different tissue types of individuals with DM and/or prediabetes. A recent systematic study of dysregulated miRNAs in T2DM
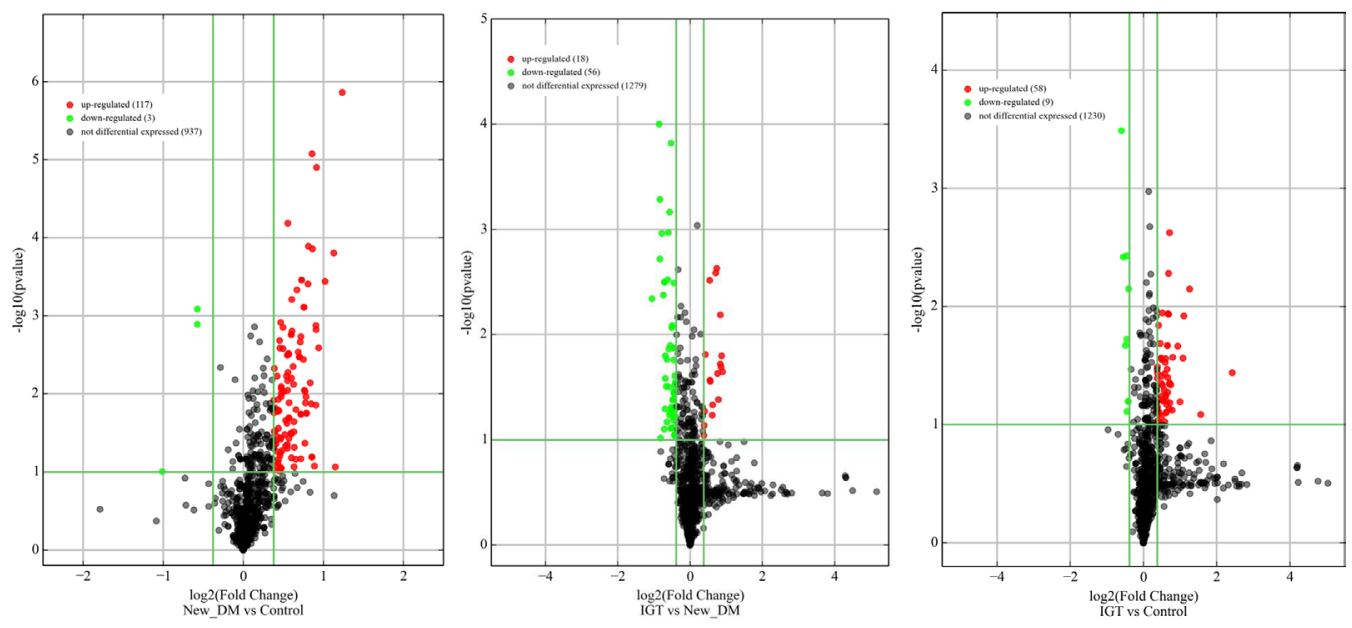

Figure 2: Volcano plots of precursor miRNA in individuals with screen-detected diabetes (DM), impaired glucose tolerance (IGT) compared to those with normal glucose tolerance (NGT). Differentially expressed miRNAs are those having fold changes $\geq 1.3$, $\mathrm{p}$-value $\leq 0.1$. 
Table 2: Dysregulated Pre miRNA and mature miRNA in screen-detected diabetes and impaired glucose tolerance versus normoglucotolerant individuals

\begin{tabular}{|c|c|c|c|c|c|c|c|}
\hline $\begin{array}{l}\text { Pre-miRNA } \\
\text { accession } \\
\text { number }\end{array}$ & Pre-miRNA & $\begin{array}{c}\text { miRNA accession } \\
\text { number }\end{array}$ & miRNA & $\begin{array}{l}\text { DM vs NGT } \\
\text { fold change }\end{array}$ & p-value & $\begin{array}{l}\text { IGT vs NGT } \\
\text { fold change }\end{array}$ & p-value \\
\hline MI0000746 & hsa-mir-99b & MIMAT0000689 & hsa-miR-99b-5p & 1,820588 & 0,000138758 & 2,959804 & 0,082063563 \\
\hline MI0000749 & hsa-mir-30e & MIMAT0000693 & hsa-miR-30e-3p & 1,784615 & 0,007252301 & 2,398601 & 0,007101456 \\
\hline MYNO2438 & hsa-mir-novel-chr2_50989 & $\begin{array}{l}\text { hsa-miR-novel- } \\
\text { chr2_50989 }\end{array}$ & $\begin{array}{l}\text { hsa-miR-novel- } \\
\text { chr2_50989 }\end{array}$ & 2,355212 & $1,37684 \mathrm{E}-06$ & 2.0 & 0,064120232 \\
\hline MI0006394 & hsa-mir-1260a & MIMAT0005911 & hsa-miR-1260a & 1,713994 & 0,01312321 & 2,151037 & 0,011997711 \\
\hline MI0016786 & hsa-mir-4443 & MIMAT0018961 & hsa-miR-4443 & 1,551667 & 0,085964281 & 2,115556 & 0,027357273 \\
\hline MI0000300 & hsa-mir-223 & MIMAT0000280 & hsa-miR-223-3p & 1,920202 & 0,002582624 & 1,678788 & 0,046035966 \\
\hline MI0014197 & hsa-mir-1260b & MIMAT0015041 & hsa-miR-1260b & 1,697833 & 0,009034611 & 1,917339 & 0,021651005 \\
\hline MI0000438 & hsa-mir-15b & MIMAT0000417 & hsa-miR-15b-5p & 1,885503 & $1,26022 \mathrm{E}-05$ & 1,409377 & 0,057613054 \\
\hline MYNO2570 & hsa-mir-novel-chr20_44211 & $\begin{array}{l}\text { hsa-miR-novel- } \\
\text { chr20_44211 }\end{array}$ & $\begin{array}{l}\text { hsa-miR-novel- } \\
\text { chr20_44211 }\end{array}$ & 1,813224 & $8,42372 \mathrm{E}-06$ & 1,60939 & 0,005237963 \\
\hline MYNO8 & hsa-mir-novel-chr7_78559 & $\begin{array}{c}\text { hsa-miR-novel- } \\
\text { chr7_78559 }\end{array}$ & $\begin{array}{c}\text { hsa-miR-novel- } \\
\text { chr7_78559 }\end{array}$ & 1,801314 & 0,013459765 & 1,535304 & 0,062496157 \\
\hline MI0000809 & hsa-mir-151a & MIMAT0004697 & $\begin{array}{l}\text { hsa-miR-151a- } \\
5 \mathrm{p}\end{array}$ & 1,705487 & 0,042803475 & 1,723404 & 0,075509343 \\
\hline MI0016746 & hsa-mir-548o-2 & MIMAT0005919 & $\begin{array}{c}\text { hsa-miR-548o- } \\
3 \mathrm{p}\end{array}$ & 1,657303 & 0,000348585 & 1,598315 & 0,011517573 \\
\hline MI0006402 & hsa-mir-548o & MIMAT0005919 & $\begin{array}{c}\text { hsa-miR-548o- } \\
3 \mathrm{p}\end{array}$ & 1,657303 & 0,000348585 & 1,598315 & 0,011517573 \\
\hline MI0003772 & hsa-mir-151b & MIMAT0010214 & hsa-miR-151b & 1,397004 & 0,053368366 & 1,58427 & 0,039862735 \\
\hline MYNO2414 & hsa-mir-novel-chr1_36178 & $\begin{array}{c}\text { hsa-miR-novel- } \\
\text { chr1_36178 }\end{array}$ & $\begin{array}{c}\text { hsa-miR-novel- } \\
\text { chr1_36178 }\end{array}$ & 1,577617 & 0,015973544 & 1,483755 & 0,051048972 \\
\hline MI0001448 & hsa-mir-425 & MIMAT0003393 & hsa-miR-425-5p & 1,545474 & 0,007580947 & 1,366328 & 0,0612974 \\
\hline MYNO2060 & hsa-mir-novel-chr5_70692 & $\begin{array}{c}\text { hsa-miR-novel- } \\
\text { chr5_70692 }\end{array}$ & $\begin{array}{c}\text { hsa-miR-novel- } \\
\text { chr5_70692 }\end{array}$ & 1,524669 & 0,001574976 & 1,405218 & 0,085501759 \\
\hline MI0000456 & hsa-mir-140 & MIMAT0004597 & hsa-miR-140-3p & 1,486817 & 0,013053757 & 1,521131 & 0,056333534 \\
\hline MI0000108 & hsa-mir-103a-2 & MIMAT0000101 & $\begin{array}{c}\text { hsa-miR-103a- } \\
3 p\end{array}$ & 1,514849 & 0,001742993 & 1,445334 & 0,06271756 \\
\hline MI0000109 & hsa-mir-103a-1 & MIMAT0000101 & $\begin{array}{c}\text { hsa-miR-103a- } \\
3 p\end{array}$ & 1,514822 & 0,001750868 & 1,445326 & 0,062651884 \\
\hline MYNO1801 & hsa-mir-novel-chr17_28671 & $\begin{array}{c}\text { hsa-miR-novel- } \\
\text { chr17_28671 }\end{array}$ & $\begin{array}{c}\text { hsa-miR-novel- } \\
\text { chr17_28671 }\end{array}$ & 1,433333 & 0,033228045 & 1,51 & 0,029705075 \\
\hline MI0000289 & hsa-mir-181a-1 & MIMAT0000256 & $\begin{array}{l}\text { hsa-miR-181a- } \\
5 \mathrm{p}\end{array}$ & 1,465751 & 0,003192238 & 1,3978 & 0,02734155 \\
\hline MI0000269 & hsa-mir-181a-2 & MIMAT0000256 & $\begin{array}{l}\text { hsa-miR-181a- } \\
5 \mathrm{p}\end{array}$ & 1,465751 & 0,003192238 & 1,3978 & 0,02734155 \\
\hline MI0016005 & hsa-mir-3615 & MIMAT0017994 & hsa-miR-3615 & 1,390032 & 0,009862178 & 1,42043 & 0,028236924 \\
\hline MYNO12 & hsa-mir-novel-chr19_33126 & $\begin{array}{c}\text { hsa-miR-novel- } \\
\text { chr19_33126 }\end{array}$ & $\begin{array}{l}\text { hsa-miR-novel- } \\
\text { chr19_33126 }\end{array}$ & 1,410758 & 0,001410185 & 1,337408 & 0,094043894 \\
\hline MI0000269 & hsa-mir-181a-2 & MIMAT0004558 & $\begin{array}{c}\text { hsa-miR-181a- } \\
2-3 p\end{array}$ & 1,34125 & 0,005944189 & 1,3025 & 0,077287593 \\
\hline
\end{tabular}

identified a total of 158 dysregulated miRNAs in adipose, islet, skeletal muscle, whole blood, PBMC, plasma and serum [26]. Similarly we found $36(23 \%)$ of these miRNAs dysregulated in T2DM and IGT (Supplementary Table 4). Furthermore, three additional miRNAs (miR27b, miR-98, and miR-21) previously reported to be dysregulated in mixed ethnic ancestry women with IGT or T2DM [27] were also differentially expressed in screen-detected DM in our sample. The miRNAs found in the current study and others have been shown to play a direct role in insulin production and secretion [21-25, 28]. This was confirmed by bioinformatics techniques we applied to identify the potential biological functions affected by the miRNA signatures. p53 signaling, PI3K/ Akt, p53 signaling and MAPK were respectively the $2^{\text {nd }}$, $3^{\text {rd }}$ and $6^{\text {th }}$ targeted significant pathways in enrichment analysis by KEGG. The PI3K/Akt/ and MAPK pathways plays a major signaling role in the cellular response to extracellular stimuli, including glucose homeostasis, cell proliferation and survival [29]. In glucose homeostasis, 


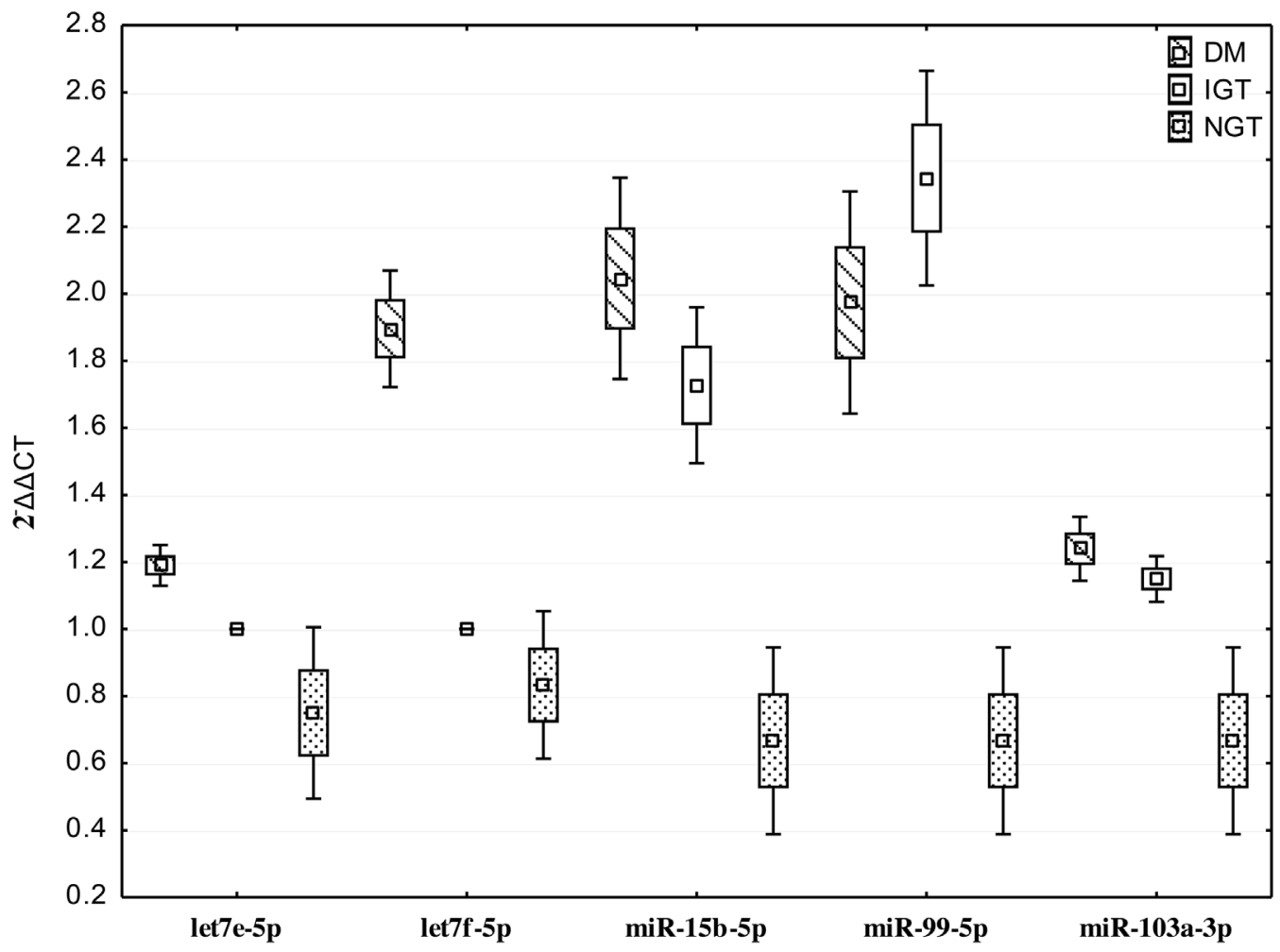

Figure 3: Quantitative real time PCR of differentially expressed miRNAs in individuals with screen-detected diabetes (DM), impaired glucose tolerance (IGT) and normol glucose tolerance (NGT). Data presented as mean and range.
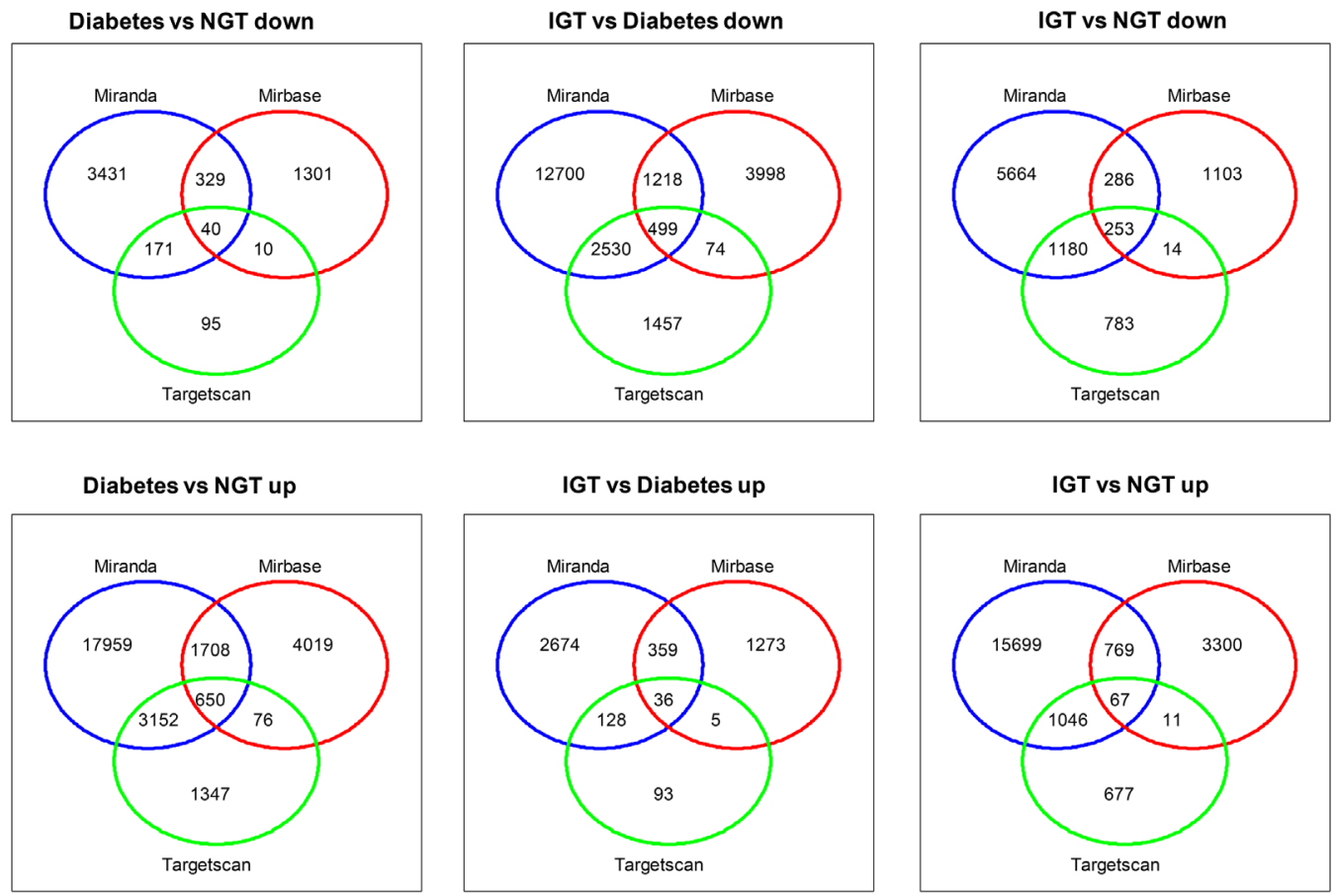

Figure 4: Venn diagrams showing target genes with three databases (TargetScan, Miranda and Microcosm v5) in individuals with screen-detected diabetes (DM), impaired glucose tolerance (IGT) compared to those with normal glucose tolerance (NGT). 
the activation of these pathways is directly under the control of insulin receptors upon insulin stimulation [30]. A number of miRNAs such as the let-7 family, 30ep-5p $[26,31,32]$ found in this study and others have been shown to be involved in these pathways. These miRNAs have be reported to exert their function by suppressing the expression of insulin receptor genes [17, 32].

Although many similarities were found between this study and others, our study is unique for uncovering that some of these miRNAs were differentially expressed between diabetic and non-diabetic dysglycaemia. Indeed, using OGTT to characterise asymptomatic participants, we identified three miRNAs that potentially distinguish between diabetic and non-diabetic hyperglycaemia. For example, miR-126-3p, and miR-28-3p were upregulated in IGT when compared to screen-detected DM, whilst miR-486-5p was down-regulated in screen-detected $\mathrm{DM}$ in comparison to either IGT or NGT. miR-126 is expressed by cells that modulate inflammatory response and vascular homeostasis through enhanced production of anti-inflammatory chemokines, and has been shown to be reduced in T2DM [33-37]. The downgrelation of miR126 has been shown to be mostly pronounced in poorly controlled T2DM and in T2DM with complications when compared to sujects with T2DM without complication [38]. Similarly, in a study that investigated miR-126 in serum of DM patients with varying degrees of retinopathy, miR-126 was reduced in patients versus the controls, but lowest in patients with proliferative diabetic retinopathy [39]. Taken together, our findings of upregulated miR126 and others in IGT versus screen-detected DM most probably point towards a cascading reduction with respect to diabetes related complications suggesting a potential role for miR-126 in distinguishing prediabetes from diabetes. Indeed, Liu et al [40]), examined the usefulness of miR-126 in predicting prediabetes and T2DM and reported lower levels in T2DM compared to prediabetes, even though both were significanlty lower than in healthy controls. It is important to note that a number of miRNAs including novel ones with potential to distinguish between hyperglycaemia and normal glucose tolerance were uncovered in the current study. For example, miR- hsa-miR-1299 had the highest fold change in IGT versus controls and was not detected in individuals with DM, whilst mir-novel-chr2_55842 was amongst the $10^{\text {th }}$ most differentially expressed in IGT only. In hepato-hepatocellular carcinoma, miR-1299 inhibits cell proliferation by targeting cyclin-dependent kinase 6, [41] however there is limited information about miR-1299 in diabetes. Therefore, further studies are needed to elucidate the molecular mechanisms of miR-1299 and other novel miRNAs identified in this study.

The major strengths of this study lies in the choice of high throughput sequencing which is a powerful strategy for identifying known and new candidate miRNAs related to the disease process. Furthermore, this method was ideal to cover unidentified miRNAs in this rather heterogeneous mixed ancestry population. Importantly, we did not include individuals on diabetes lowering treatment as this had the potential to bias our findings. Metformin, a drug that targets protein kinase activity and which is widely used for the treatment of insulin resistance, is believed to operate mainly by activating the insulin signaling pathway AMPK [29]. Lastly, our study participants were well-characterized by OGTT, and were matched for age, sex, blood pressure, serum cotinine and cholesterol. Therefore, we were able to uncover several known and novel miRNAs showing statistically significant evidence for differential expression by glucose tolerance status. In total we identified 57 novel differentially expressed miRNAs of which miR-novel-chr2_50989 deserves further consideration as this was amongst the top differentially expressed in both screen-detected and IGT. Despite these strengths, our study has limitations. Although our sample size is comparable to similar studies, we still consider it small. A small sample size has the potential to limit the ability to detect some significant differences in miRNA expression levels. Although, we employed RT-qPCR to confirm the expression of miRNAs identified by the deep sequencing approach, this was not performed in an independent sample. Therefore, a larger independent study is required to confirm our findings in this population. The cross-sectional design of this study limits our ability to draw conclusions with regards to association with disease progression, or to explore the role of particularly novel miRNAS in the molecular mechanisms of hyperglycaemia. Despite these considerations, several miRNAs whose molecular mechanism has been previously investigated were also found in this population group.

Overall, in addition to complementing earlier studies on miRNAs in prediabetes and diabetes, our findings provide evidence of known and novel differentially expressed miRNAs in African mixed ancestry individuals with IGT and screen-detected DM. We further observed that the aberrant expression profiles of miRNAs were linked to several biological processes, such as signal transduction, cell-cell communications, cell growth and death, immune response, endocrine system and metabolic diseases. Larger prospective studies in this and other racial populations from Africa are needed to characterize the molecular mechanisms of African-specific differentially expressed miRNAs, as well as assess their potential to predict worsening of glucose tolerance status.

\section{MATERIALS AND METHODS}

\section{Ethical approval of the study}

This investigation is based on the Cape Town Vascular and Metabolic Health (VMH) study, that has been approved by the Research Ethics Committees of the Cape Peninsula University of Technology (CPUT) and 
Stellenbosch University (respectively, NHREC: REC $230408-014$ and N14/01/003). For this sub-study, ethical approval was also obtained from the CPUT Health and Wellness Sciences Research Ethics Committee (CPUT/ HW-REC 2014/H08). The study was conducted according to the Code of Ethics of the World Medical Association (Declaration of Helsinki). All participants signed written informed consent after all the procedures had been fully explained in the language of their choice.

\section{Study design and procedures}

This was a cross-sectional study involving participants from the ongoing Cape Town Vascular and Metabolic Health (VMH) study. VMH is an extension of the Cape Town Bellville South study, which has been described in details previously [42, 43]. Data collection was based on a standardised questionnaire available in the electronic form on a password-protected personal digital assistant (PDA). Upon completion of the assessment of each participant, data were automatically encrypted and transmitted via mobile internet connection to a dedicated server, from which they were checked for completion, downloaded and stored for future use. Physical examination involved data collection on blood pressure (BP) which was measured according to the World Health Organisation (WHO) guidelines [44], using a semi-automatic digital blood pressure monitor (Omron M6 comfort-preformed cuff BP Monitor) on the right arm in sitting position and at rest for at least 10 minutes. Body weight (to the nearest $0.1 \mathrm{~kg}$ ) was measured with the subject in light clothing and without shoes, using an Omron body fat meter HBF-511digital bathroom scale, which was calibrated and standardized using a weight of known mass. Height to the nearest centimetre was measured with a stadiometer, with subjects standing on a flat surface. Body Mass Index (BMI) was calculated as weight per square meter $\left(\mathrm{kg} / \mathrm{m}^{2}\right)$. Waist circumference was measured with a non-elastic tape at the level of the narrowest part of the torso, as seen from the anterior view. BP and anthropometric measurements were performed three times and their average used for analysis.

All participants underwent a $75 \mathrm{~g}$ oral glucose tolerance test (OGTT) as recommended by the WHO [20]. Further, the following biochemical parameters were analyzed at an ISO 15189 accredited Pathology practice (PathCare, Reference Laboratory, Cape Town, South Africa): glycated haemoglobin (HbAlc) by High Perfomance Liquid Chromotography (Biorad Variant Turbo, BioRad, South Africa); serum insulin by a paramagnetic particle chemiluminescence assay (Beckman DXI, Beckman Coulter, South Africa); serum cotinine by Competitive Chemiluminescent (Immulite 2000, Siemens, South Africa); plasma glucose by enzymatic hexokinase method (Beckman AU, Beckman Coulter, South Africa); total cholesterol (TC); high density lipoprotein cholesterol (HDL-c) by enzymatic immunoinhibition - End Point (Beckman AU, Beckman Coulter, South Africa); triglycerides (TG) by glycerol phosphate oxidase-peroxidase, End Point (Beckman AU, Beckman Coulter, South Africa); low density lipoprotein cholesterol (LDL) by enzymatic selective protection - End Point (Beckman AU, Beckman Coulter, South Africa); and ultrasensitive C-reactive protein (CRP) by Latex Particle immunoturbidimetry (Beckman AU, Beckman Coulter, South Africa). In addition, blood sample were collected in a Tempus RNA tube (Applied Biosystems) and stored at -80 degrees for RNA extraction and analysis. Participants selected for this analysis were 12 individuals with screendetected diabetes, 12 with prediabetes and 12 with normal glucose tolerance, matched for age, blood pressure, smoking and body mass index.

\section{RNA isolation}

Total RNA, including miRNA, was isolated from whole blood using MagMax total RNA isolation kit (ThermoFisher Scientific, South Africa) according to manufacture's instructions. The concentration and purity of the RNA samples were determined using a NanoDrop ND-1000 spectrophotometer and RNA samples that met a $260 / 280$ value $>1.8$ and a concentration $>20 \mathrm{ug} / \mathrm{ul}$ were used for miRNA sequencing. Furthermore, RNA integrity was evaluated by denaturing agarose gel electrophoresis.

\section{miRNA sequencing}

Small RNA library construction, deep sequencing, and data processing were performed at Arraystar Inc., Rockville, USA. Total RNA of each sample was used to prepare the miRNA sequencing library which included the following steps: 1) 3'-adapter ligation with T4 RNA ligase 2 (truncated); 2) 5'-adapter ligation with T4 RNA ligase; 3) cDNA synthesis with RT primer; 4) PCR amplification; 5) extraction and purification of $\sim 130-150 \mathrm{bp}$ PCR amplified fragments (correspond to $\sim 15-35$ nt small RNAs) from the PAGE gel. After the completed libraries were quantified with Agilent 2100 Bioanalyzer, the DNA fragments in the libraries were denatured with $0.1 \mathrm{M} \mathrm{NaOH}$ to generate single-stranded DNA molecules, captured on Illumina flow cells, amplified in situ and finally sequenced for 51 cycles on Illumina HiSeq according to the manufacturer's instruction. Raw sequences were generated as clean reads from Illumina HiSeq by real-time base calling and quality filtering. The clean reads that passed the quality filter were processed to remove the adaptor sequence as the trimmed reads. The trimmed reads (length $\geq 15 \mathrm{nt}$ ) were aligned to the human pre-miRNA in miRBase 21 , using novoalign software. The miRNA expression levels were measured and normalized as transcripts per million of total aligned miRNA reads (TPM). miRNAs having fold changes $>=$ 1.3 , P-value $<=0.1$ were selected as the differentially expressed miRNAs. Novel miRNAs were predicted by algorithms such as miRDeep [45]. 


\section{Analysis of individual miRNAs using quantitative reverse-transcription PCR (RT- qPCR)}

To confirm the expression of miRNAs identified by the deep sequencing approach, RT-qPCR analysis was performed using miRNA from the same samples used in miRNA deep sequencing. miRNA was converted to cDNA using the TaqMan MicroRNA Reverse Transcription Kit according to the manufacturer's protocol (Life Technologies, USA). miRNA expression levels were assessed using and TaqMan miRNA Assay primers in conjuction with Quantum Studio 7 (Life Technologies, USA). The delta delta $\mathrm{Ct}\left(2^{-\Delta \Delta C T}\right)$ method was used to determine fold-change of miRNA expression between samples using the average expression of RNU6B, and miR-425 as endogenous controls. We selected the following miRNA for validation, let7e-5p, let7f-5p, miR15b-5p, miR-99b-5p, miR-103a-3p.

\section{Target prediction and functional enrichment analysis}

To improve the accuracy of messenger RNA (mRNA) gene targets, we used three different prediction algorithms, TargetScan (v6.2) (http://www.targetscan. org/vert_60/), Miranda, and Microcosm v5. The Venny tool (Venny v2.0.2) (http://bioinfogp.cnb.csic.es) was used to filter miRNA gene targets common to all three programs. miRNAs with the highest fold changes among the increased and decreased miRNAs were chosen for mRNA network analysis. These networks showed how one miRNA targets several mRNAs, which in turn can be targeted by several miRNAs and these are shown in Supplementary Figure 1. Commonly predicted gene targets were subjected to functional analysis using Kyoto Encyclopedia of Genes and Genomes (KEGG). We used a conservative Fisher's exact test and the false discovery rate method to calculate the targeted pathways.

\section{Statistical analysis}

Data were analysed using the $\mathrm{R}$ statistical software version 3.2.2 [2015-08-14], (The R Foundation for Statistical Computing, Vienna, Austria). Variables are summarized as mean and standard deviation or median $\left[25^{\text {th }}-75^{\text {th }}\right.$ percentiles $)$. The Shapiro-Wilk W test was employed to determine whether the data were normally distributed, based on probability thresholds of $\mathrm{p}>0.1$.

\section{Author contributions}

TEM: conception and design of the study, acquisition of data, or analysis and interpretation of data; drafting of the article or revising it for important content; final approval of the version to be published; responsible for ensuring that all authors have agreed 1) to be authors and to be listed in the order specified by the submitting author; 2) to the manuscript's content; and 3) to its submission to the journal. APK: analysis and interpretation of data; revising it for important content; final approval of the version to be published. SH: analysis and interpretation of data; revising it for important content; final approval of the version to be published DLM: analysis and interpretation of data; final approval of the version to be published. YYY: revising it for important content; final approval of the version to be published. RTE: conception and design of the study revising it for important content; final approval of the version to be published.

\section{ACKNOWLEDGMENTS}

We thank the Bellville South (Ward 009) community for participating in the study. We are also grateful to the Bellville South community Health Forum for supporting the engagement with the Bellville South community.

\section{CONFLICTS OF INTEREST}

The authors declare no conflicts of interest.

\section{FUNDING}

This research project was funded by the South African Medical Research Council (SAMRC) with funds from National Treasury under its Economic Competitiveness and Support Package (MRC-RFAUFSP-01-2013/ VMH Study) and strategic funds from the SAMRC received from the South African National Department of Health. Any opinion, finding and conclusion or recommendation expressed in this material is that of the author(s) and the MRC does not accept any liability in this regard.

\section{REFERENCES}

1. International Diabetes Federation. IDF Diabetes Atlas, 7th Edition. 7th edition ed. Brussels: International Diabetes Federation; 2015.

2. NCD Risk Factor Collaboration (NCD-RisC) - Africa Working Group. Trends in obesity and diabetes across Africa from 1980 to 2014: an analysis of pooled populationbased studies. Int J Epidemiol. 2017; 46:1421-1432.

3. Shaw JE, Sicree RA, Zimmet PZ. Global estimates of the prevalence of diabetes for 2010 and 2030. Diabetes Res Clin Pract. 2010;87:4-14.

4. Tabak AG, Herder C, Rathmann W, Brunner EJ, Kivimaki M. Prediabetes: a high-risk state for diabetes development. Lancet. 2012;379:2279-90. 
5. Hopper I, Billah B, Skiba M, Krum H. Prevention of diabetes and reduction in major cardiovascular events in studies of subjects with prediabetes: meta-analysis of randomised controlled clinical trials. Eur J Cardiovasc Prev Rehabil. 2011;18:813-23.

6. Perreault L, Pan Q, Mather KJ, Watson KE, Hamman RF, Kahn SE; Diabetes Prevention Program Research Group. Effect of regression from prediabetes to normal glucose regulation on long-term reduction in diabetes risk: results from the Diabetes Prevention Program Outcomes Study. Lancet. 2012;379:2243-51.

7. Chen X, Ba Y, Ma L, Cai X, Yin Y, Wang K, Guo J, Zhang Y, Chen J, Guo X, Li Q, Li X, Wang W, et al. Characterization of microRNAs in serum: a novel class of biomarkers for diagnosis of cancer and other diseases. Cell Res. 2008;18:997-1006.

8. Price NL, Ramirez CM, Fernandez-Hernando C. Relevance of microRNA in metabolic diseases. Crit Rev Clin Lab Sci. 2014;51:305-20.

9. Chen H, Lan HY, Roukos DH, Cho WC. Application of microRNAs in diabetes mellitus. J Endocrinol. 2014;222:R1-R10.

10. miRBase Griffiths-Jones Lab. [cited 17.07.2017]. Available from: http://www.mirbase.org/cgi-bin/mirna_summary. $\mathrm{pl}$ ?org $=$ hsa.

11. Winter J, Jung S, Keller S, Gregory RI, Diederichs S. Many roads to maturity: microRNA biogenesis pathways and their regulation. Nat Cell Biol. 2009;11:228-34.

12. Kim VN, Han J, Siomi MC. Biogenesis of small RNAs in animals. Nat Rev Mol Cell Biol. 2009;10:126-39.

13. Fabian MR, Sonenberg N, Filipowicz W. Regulation of mRNA translation and stability by microRNAs. Annu Rev Biochem. 2010;79:351-79.

14. Caputa G, Schaffer JE. RNA Regulation of Lipotoxicity and Metabolic Stress. Diabetes. 2016;65:1816-23.

15. Guay C, Regazzi R. Role of islet microRNAs in diabetes: which model for which question? Diabetologia. 2015;58:456-63.

16. Wang X, Sundquist J, Zöller B, Memon AA, Palmér K, Sundquist K, Bennet L. Determination of 14 circulating microRNAs in Swedes and Iraqis with and without diabetes mellitus type 2. PLoS One. 2014; 9:e86792.

17. Karolina DS, Armugam A, Tavintharan S, Wong MT, Lim SC, Sum CF, Jeyaseelan K. MicroRNA 144 impairs insulin signaling by inhibiting the expression of insulin receptor substrate 1 in type 2 diabetes mellitus. PLoS One. 2011;6:e22839.

18. Erasmus RT, Soita DJ, Hassan MS, Blanco-Blanco E, Vergotine Z, Kengne AP, Matsha TE. High prevalence of diabetes mellitus and metabolic syndrome in a South African mixed ancestry population: the Bellville-South Africa study - Baseline data. S Afr Med J. 2012;102:841-4.

19. Ko GT, Chan JC, Woo J, Lau E, Yeung VT, Chow CC, Cockram CS. The reproducibility and usefulness of the oral glucose tolerance test in screening for diabetes and other cardiovascular risk factors. Ann Clin Biochem. 1998; 35:62-7.

20. Alberti KG, Zimmet P. Definition, diagnosis and classification of diabetes mellitus and its complications. Part 1: diagnosis and classification of diabetes mellitus provisional report of a WHO consultation. Diabet Med. 1998;15:539-53.

21. Zhu H, Shyh-Chang N, Segre AV, Shinoda G, Shah SP, Einhorn WS, Takeuchi A, Engreitz JM, Hagan JP, Kharas MG, Urbach A, Thornton JE, Triboulet R, et al. The Lin28/let-7 axis regulates glucose metabolism. Cell. 2011;147:81-94.

22. Gu N, You L, Shi C, Yang L, Pang L, Cui X, Ji C, Zheng W, Guo X. Expression of miR-199a-3p in human adipocytes is regulated by free fatty acids and adipokines. Mol Med Rep. 2016;14:1180-6.

23. Poy MN, Eliasson L, Krutzfeldt J, Kuwajima S, Ma X, Macdonald PE, Pfeffer S, Tuschl T, Rajewsky N, Rorsman $\mathrm{P}$, Stoffel M. A pancreatic islet-specific microRNA regulates insulin secretion. Nature. 2004;432:226-30.

24. Trajkovski M, Hausser J, Soutschek J, Bhat B, Akin A, Zavolan M, Heim MH, Stoffel M. MicroRNAs 103 and 107 regulate insulin sensitivity. Nature. 2011;474:649-53.

25. Hashimoto N, Kido Y, Uchida T, Asahara S, Shigeyama Y, Matsuda T, Takeda A, Tsuchihashi D, Nishizawa A, Ogawa W, Fujimoto Y, Okamura H, Arden KC, et al. Ablation of PDK1 in pancreatic beta cells induces diabetes as a result of loss of beta cell mass. Nat Genet. 2006;38:589-93.

26. He Y, Ding Y, Liang B, Lin J, Kim TK, Yu H, Hang H, Wang K. A Systematic Study of Dysregulated MicroRNA in Type 2 Diabetes Mellitus. Int J Mol Sci. 2017:18.

27. Dias S, Hemmings S, Muller C, Louw J, Pheiffer C. MicroRNA Expression Varies according to Glucose Tolerance, Measurement Platform, and Biological Source. Biomed Res Int. 2017;2017:1080157.

28. El Ouaamari A, Baroukh N, Martens GA, Lebrun P, Pipeleers D, van Obberghen E. miR-375 targets 3'-phosphoinositide-dependent protein kinase-1 and regulates glucose-induced biological responses in pancreatic beta-cells. Diabetes. 2008;57:2708-17.

29. Schultze SM, Hemmings BA, Niessen M, Tschopp O. PI3K/AKT, MAPK and AMPK signalling: protein kinases in glucose homeostasis. Expert Rev Mol Med. 2012;14:e1.

30. White MF. IRS proteins and the common path to diabetes. Am J Physiol Endocrinol Metab. 2002;283:E413-22.

31. Erener S, Marwaha A, Tan R, Panagiotopoulos C, Kieffer TJ. Profiling of circulating microRNAs in children with recent onset of type 1 diabetes. JCI Insight. 2017;2:e89656.

32. Plaisance V, Waeber G, Regazzi R, Abderrahmani A. Role of microRNAs in islet beta-cell compensation and failure during diabetes. J Diabetes Res. 2014;2014:618652.

33. Tang ST, Wang F, Shao M, Wang Y, Zhu HQ. MicroRNA-126 suppresses inflammation in endothelial 
cells under hyperglycemic condition by targeting HMGB1. Vascul Pharmacol. 2017;88:48-55.

34. Wu Y, Song LT, Li JS, Zhu DW, Jiang SY, Deng JY. MicroRNA-126 Regulates Inflammatory Cytokine Secretion in Human Gingival Fibroblasts Under High Glucose via Targeting Tumor Necrosis Factor Receptor Associated Factor 6. J Periodontol. 2017; 88:e179-e187.

35. Ishizaki T, Tamiya $\mathrm{T}$, Taniguchi K, Morita R, Kato R, Okamoto F, Saeki K, Nomura M, Nojima Y, Yoshimura A. miR126 positively regulates mast cell proliferation and cytokine production through suppressing Spred1. Genes Cells. 2011;16:803-14.

36. Zhu H, Leung SW. Identification of microRNA biomarkers in type 2 diabetes: a meta-analysis of controlled profiling studies. Diabetologia. 2015;58:900-11.

37. Zampetaki A, Kiechl S, Drozdov I, Willeit P, Mayr U, Prokopi M, Mayr A, Weger S, Oberhollenzer F, Bonora E, Shah A, Willeit J, Mayr M. Plasma microRNA profiling reveals loss of endothelial miR-126 and other microRNAs in type 2 diabetes. Circ Res. 2010;107:810-7.

38. Zhang T, Lv C, Li L, Chen S, Liu S, Wang C, Su B. Plasma miR-126 is a potential biomarker for early prediction of type 2 diabetes mellitus in susceptible individuals. Biomed Res Int. 2013;2013:761617.

39. Qin LL, An MX, Liu YL, Xu HC, Lu ZQ. MicroRNA-126: a promising novel biomarker in peripheral blood for diabetic retinopathy. Int J Ophthalmol. 2017;10:530-4.
40. Liu Y, Gao G, Yang C, Zhou K, Shen B, Liang H, Jiang X. The role of circulating microRNA-126 (miR-126): a novel biomarker for screening prediabetes and newly diagnosed type 2 diabetes mellitus. Int J Mol Sci. 2014;15:10567-77.

41. Zhu H, Wang G, Zhou X, Song X, Gao H, Ma C, Chang H, Li H, Liu FF, Lu J, Ma J. miR-1299 suppresses cell proliferation of hepatocellular carcinoma (HCC) by targeting CDK6. Biomed Pharmacother. 2016;83:792-7.

42. Kengne AP, Erasmus RT, Levitt NS, Matsha TE. Alternative indices of glucose homeostasis as biochemical diagnostic tests for abnormal glucose tolerance in an African setting. Prim Care Diabetes. 2017;11:119-31.

43. Matsha TE, Hassan MS, Kidd M, Erasmus RT. The 30-year cardiovascular risk profile of South Africans with diagnosed diabetes, undiagnosed diabetes, pre-diabetes or normoglycaemia: the Bellville, South Africa pilot study. Cardiovasc J Afr. 2012;23:5-11.

44. Chalmers J, MacMahon S, Mancia G, Whitworth J, Beilin L, Hansson L, Neal B, Rodgers A, Ni Mhurchu C, Clark T. 1999 World Health Organization-International Society of Hypertension Guidelines for the management of hypertension. Guidelines sub-committee of the World Health Organization. Clin Exp Hypertens. 1999;21:1009-60

45. Friedlander MR, Chen W, Adamidi C, Maaskola J, Einspanier R, Knespel S, Rajewsky N. Discovering microRNAs from deep sequencing data using miRDeep. Nat Biotechnol. 2008;26:407-15. 